\title{
Clinical surveillance for human astrovirus in Monastir region, Tunisia
}

\author{
Abir Monastiri ${ }^{*}$, Mahjoub Aouni ${ }^{1}$, Susana Guix ${ }^{2}$, Badereddine Mechri ${ }^{1}$, Marc Lopez-Roig ${ }^{3}$, Nabil Ben Salem Abid ${ }^{1}$, \\ Neji Gueddiche ${ }^{4}$, Sabeur Hamami ${ }^{4}$, Lamjed Boughzala ${ }^{1}$ and Jordi Serra-Cobo ${ }^{3}$
}

\begin{abstract}
Background/aims: Astroviruses (AstVs) are enteric viruses that can cause gastroenteritis in children. This study is part of monitoring the circulation of astroviruses in children hospitalized and/or outpatients for acute gastroenteritis at the primary care center of Ouerdanine or at the Pediatric Department of the University Hospital Fattouma-Bourguiba (Monastir, Tunisia). The aims of our study were to know the prevalence of human astrovirus in clinical samples of children, characterize the strains and evaluate the infectivity of isolated strains on cell culture.

Methods: Fifty stool samples were collected from children under five years old in the region of Monastir (Tunisia) from October 2010 to June 2011. All specimens were subjected to RT-PCR amplification followed by sequencing and phylogenetic analysis.

Results: The study shows a low prevalence of astrovirus (4 \%) in children. The two positive samples obtained were HAstV type 3. Samples that were RT-PCR positive were cultured in CaCO-2 cells and the presence of infectious viral particles was confirmed. The phylogenetic analysis shows that the different HAstV-3 strains isolated in Tunisia are grouped into two clusters. The first cluster includes strains obtained in 2004, which belong to lineage HAstV-3a, while strains isolated in 2010 belong to lineage HAstV-3c.

Conclusions: This study is part of monitoring the circulation of astroviruses in children younger than five years old from Monastir region, Tunisia. The results show low prevalence (4\%). All genotyped samples belonged to lineage HAstV-3c, which could be presently emerging. Two different lineages have been isolated in Tunisia: HAstV-3a in 2004 and HAstV-3C in 2010.
\end{abstract}

Keywords: Human astrovirus, Clinical surveillance, RT-PCR, Cell culture, Tunisia

\section{Background}

Astroviruses (AstVs) are enteric viruses that can cause gastroenteritis in children and a severe disease in immunocompromised and elderly people $[1,2]$. The prevalence rate of human astrovirus (HAstV) infection ranged from $2 \%$ to $9 \%$ among children with diarrhea, although incidences over $60 \%$ have also been reported [3]. The morbidity varies depending on the season, with higher infection during the winter in temperate climates and the rainy season in tropical regions [4]. The main symptom of astrovirus infection is watery diarrhea, which is

\footnotetext{
* Correspondence: abirmonastiri@gmail.com

${ }^{1}$ Laboratory of Contagious Diseases and Biologically Active Substances, LR99ES27, Faculty of Pharmacy, University of Monastir, Avicenne Street 5000, Monastir, Tunisia

Full list of author information is available at the end of the article
}

often associated with vomiting, fever, and abdominal pain [2].

Astroviruses are a non-enveloped positive-strand RNA viruses belonging to the Astroviridae family [5]. HAstVs are genetically and antigenetically heterogeneous and include three major groups [3]. The first includes the classic eight serotypes of HAstVs (HAstV-1 to HAstV-8). The other two groups (MLB and VA clades), identified in 2009, are also prevalent but their pathogenic role in humans requires further characterization [3]. Epidemiological surveys on classic HAstVs show that HAstV-1 is the most common serotype identified worldwide in children $[6,7]$, while HAstV-6, and -7 are less frequent $[8,9]$. A precedent study, performed in Tunisia, showed the predominance of genotype HAstV-1 [10]. 
The aims of our study were to know the prevalence of HAstVs in clinical samples of children from Tunisia obtained in 2010-2011, characterize the strains and evaluate the infectivity of isolated strains.

\section{Methods}

\section{Clinical samples}

Fifty stool samples were collected from children younger than five years old hospitalized and/or seeking medical care for acute gastroenteritis at the Primary Care Center of Ouerdanine or at the Pediatric Department of the University Hospital Fattouma-Bourguiba (Monastir, Tunisia). Samples were obtained from October 2010 to June 2011 and within $24 \mathrm{~h}$ following hospitalization or visit. The main clinical symptoms of the patients were: diarrhea, fever $\left(>37^{\circ} \mathrm{C}\right)$ and vomiting. All samples were free from bacterial and parasitic enteric pathogens (tested by routine hospital procedure).

The study protocol was approved by the Ethics and Research Committee of University Hospital FattoumaBourguiba (Monastir, Tunisia) and written informed consent was obtained from the parents of the 50 study participants.

\section{Virus concentration}

Stools were suspended $(10 \%, \mathrm{w} / \mathrm{v})$ in phosphate-buffered saline (PBS) containing $2 \mathrm{M} \mathrm{NaNO}_{3}, 1 \%$ bovine serum albumin; fraction V, and $0.1 \%$ Triton X- 100 (pH 7.2). After vortexing samples were centrifuged for $15 \mathrm{~min}$ at 12,000 $\mathrm{rpm}$ and the supernatant was stored at $-70{ }^{\circ} \mathrm{C}$.

\section{Detection of Astrovirus RNA}

RNA was extracted from $400 \mu$ of stool suspension using phenol/chloroform and ethanol elution method. RNA was eluted in $30 \mu \mathrm{l}$ of DEPC-treated water (Invitrogen). RT-PCR was carried out using primers Mon269 and Mon270 [11] targeting a $449 \mathrm{bp}$ region of the capsid protein gene (ORF2), whereas primers AV1 and AV2 were used to amplify a $90 \mathrm{bp}$ highly conserved region at the 3 ' end of astrovirus genome [12]. The reverse transcription step was performed by using $5 \mu \mathrm{l}$ of RNA added to $10 \mu \mathrm{M}$ of primer Mon270, $1 \mathrm{mM}$ of each dNTP, 2 mM DTT and 1.25 U of M-MLV reverse transcriptase (Invitrogen, Tunisia) in a $25 \mu \mathrm{l}$ total reaction volume containing $25 \mathrm{mM}$ Tris- $\mathrm{HCl}(\mathrm{pH} 8.3), 20 \mathrm{mM}$ $\mathrm{KCl}$ and $3 \mathrm{mM} \mathrm{MgCl}_{2}$. The cDNA was synthesised at 42 ${ }^{\circ} \mathrm{C}$ for $60 \mathrm{~min}$. For the PCR reaction, $5 \mu \mathrm{l}$ of the RT product was added to $20 \mu \mathrm{l}$ reaction mixture containing $2 \mathrm{mM} \mathrm{MgCl}_{2}, 10 \mathrm{mM}$ of each dNTP, $10 \mu \mathrm{M}$ of each primer Mon269 and Mon270 and 1.25 U of Taq polymerase (Biomatik, Tunisia). The $25 \mu \mathrm{l}$ reaction mixture was amplified using the following cycling conditions: 40 cycles of amplification at $94{ }^{\circ} \mathrm{C}$ for $1 \mathrm{~min}$, at $50{ }^{\circ} \mathrm{C}$ for $1 \mathrm{~min}$ and at $72{ }^{\circ} \mathrm{C}$ for $1 \mathrm{~min}$. For the second RT-PCR using primers set AV1/AV2, the reaction mixture was the same as the first reaction but with the following amplification conditions: 30 cycles at $90{ }^{\circ} \mathrm{C}$ for $1 \mathrm{~min}$, at $40{ }^{\circ} \mathrm{C}$ for $2 \mathrm{~min}$ and at $72{ }^{\circ} \mathrm{C}$ for $1 \mathrm{~min}$. A total of $10 \mu \mathrm{l}$ of RT-PCR product was analyzed on a $2 \%$ agarose gel. Gel images were recorded with the Gel Doc 2000 system (Bio-Rad Laboratories). PCR products were purified using ExoASP-IT ${ }^{\circ}$ (Affymetrix) and sequenced using the ABI BigDye ${ }^{\curvearrowleft}$ Terminator v3.1 Cycle Sequencing Kit (Applied Biosystems) and ABI 3730xl DNA Analyzer (Applied Biosystems).

\section{Phylogenetic analysis}

The sequences obtained were blasted against Genbank using Sequencher 10.1 software. A phylogenetic tree was constructed with Kimura-two parameter model [13] and neighbor-joining methods (5,000 bootstrap replications) using Mega 6 software [14].

\section{Cell culture and inoculation}

CaCO-2 cell were grown in DMEM-F12 medium supplemented with $2 \mathrm{mM}$ glutamine, $10 \%$ fetal bovine serum (FBS), and $1 \%$ antibiotic mixture (100 units Penicillin $\mathrm{ml}^{-1} / 100 \mathrm{mg}$ Streptomycin $\mathrm{ml}^{-1}$ ). For inoculation, stool suspensions $(10 \%, \mathrm{v} / \mathrm{v})$ were prepared in DMEMF12 supplemented with $1 \%$ antibiotic mixture. Suspensions were kept at $4{ }^{\circ} \mathrm{C}$ for $24 \mathrm{~h}$, centrifuged for $30 \mathrm{~min}$ at 7,000 rpm and filtered $(0.22 \mu \mathrm{m}$ filter $)$. Prior to inoculation, cells were washed with serum-free DMEM-F12 and infected with the virus in the presence of $10 \mu \mathrm{g} / \mathrm{ml}$ of trypsin. After $1 \mathrm{~h}$, inoculums was replaced with fresh DMEM-F12 containing $2 \%$ FBS. RNA extraction was performed from $200 \mu \mathrm{l}$ of culture supernatant harvested at different times post-infection.

\section{Results}

\section{Astrovirus detection and typing}

We analyzed samples from 19 girls and 31 boys aged to 2 and 60 months (average 14.16 \pm 13.69 months) that came from 10 different localities (Fig. 1). Twenty-eight children were outpatients and 22 inpatients. Two samples (4\%), obtained in October 2010 from two inpatient boys aged 3 and 6 months that came from Ksar Hellal and Khniss localities, respectively, were positive (Fig. 2). The boy aged 3 months showed only diarrhea and the first symptoms appeared two days before the visit to the doctor. The patient aged 6 months showed diarrhea associated with fever $\left(39^{\circ} \mathrm{C}\right)$, vomiting and the symptoms appeared one day before the visit to the hospital.

The two sequences obtained from ORF2 belonged to lineage HAstV-3c [15]. They shared $98.6 \%$ and $97.9 \%$ of nucleotide identity with the HAstV-3c reference strains KC896091 and AB551378 isolated in Pakistan and India in 2009, respectively. Our sequences shared $92.2 \%$ of homology with the sequences of HAstV-3 obtained in 


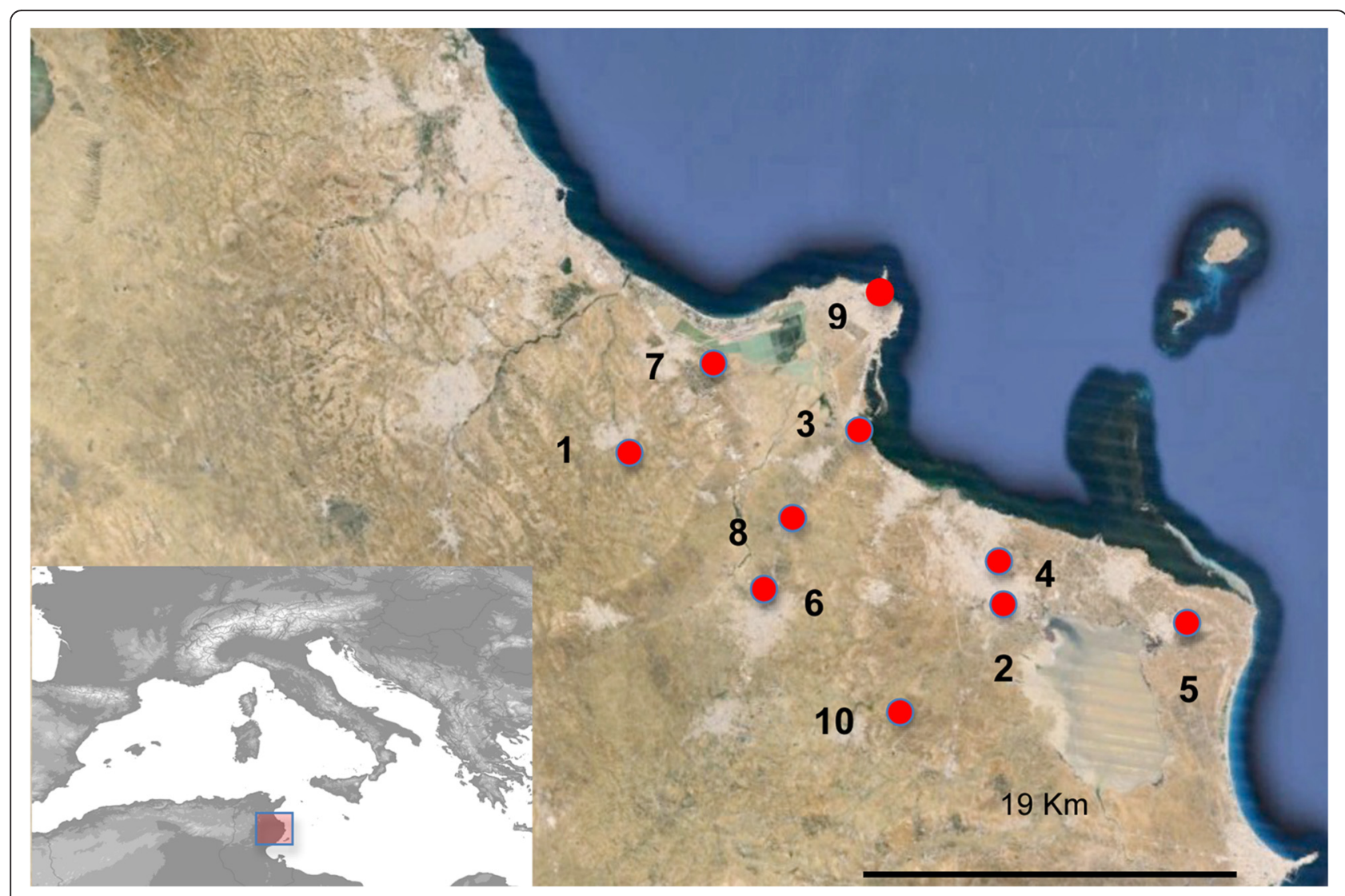

Fig. 1 Geographic distribution of infantile gastroenteritis cases in the region of Monastir (Tunisia). 1: Ouerdanine; 2: Moknine; 3: Khniss; 4: Ksar Hellal; 5: Bekalta; 6: Jemmal; 7: Sahline; 8: Menzel Ennour; 9: Monastir; 10: Beni Hassen

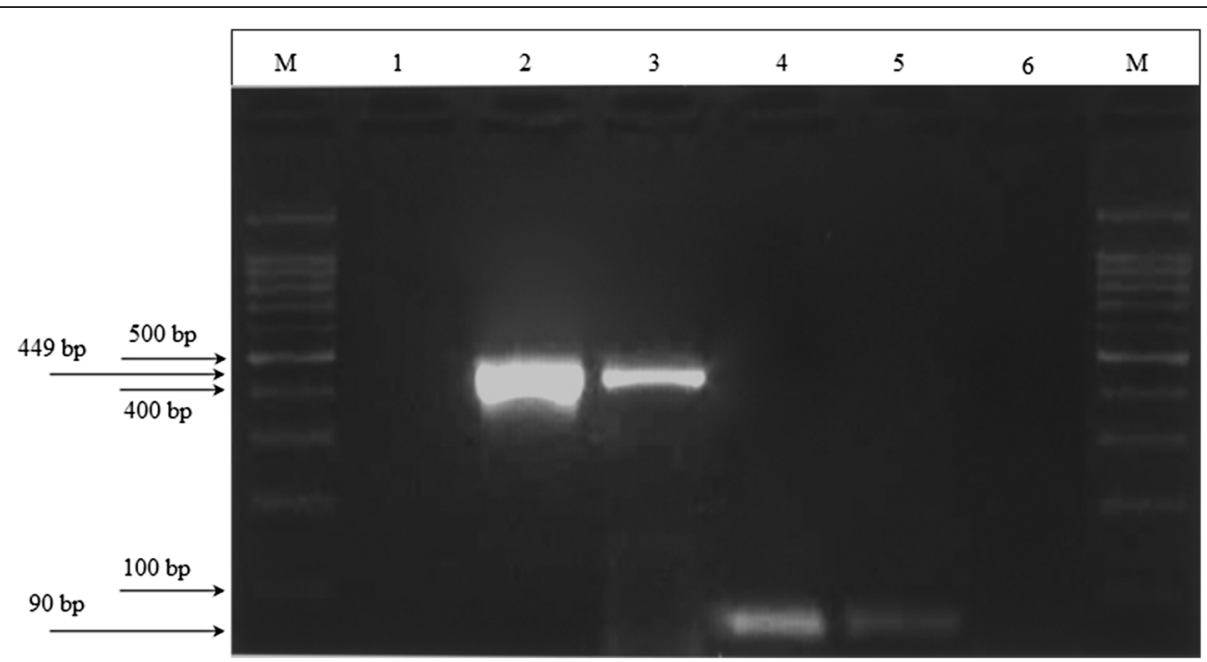

Fig. 2 Detection of astrovirus RNA in two positive stool samples. M: DNA marker 100 bp; Lane 1: Mon primers negative control (DEPC-treated water); Lane 2 and 3: PCR results of stool samples Tunisia 6 and Tunisia 7 using Mon269/Mon270 primers; Lane 4 and 5: PCR results of stool samples Tunisia 6 and Tunisia 7 using AV1/AV2 primers; Lane 6: AV primers negative control (DEPC-treated water); M: DNA marker 100 bp 
2004 at Tunisia (FJ905431, FJ905432S) [16] (Fig. 3). Sequences obtained were submitted under accession numbers: [GenBank: JQ045382, GenBank: JQ045383].

\section{Astrovirus passage in CaCO-2 cells combined to RT-PCR}

The two positive samples by RT-PCR were cultured in CaCO-2 cells and the presence of infectious viral particles was confirmed. RNA extracted from culture supernatant harvested at different times post-infection was amplified by RT-PCR using primers Mon269/Mon270. RT-PCR band intensity increased over time, which could suggest virus propagation in the infected monolayers.

\section{Discussion}

We report the results of a clinical surveillance in children hospitalized and/or outpatients for acute gastroenteritis in the Monastir region (Tunisia). This study was performed three years and half after the last clinical surveillance in the region [16]. The low prevalence of astrovirus obtained in our study (4\%) is consistent with the study conducted in the Monastir region from January 2003 to April 2007 (3.6\%) [17], as well as in other countries such as in France (6\%), Italy (3.1\%), Spain (4.9\%) and India (5.8\%) [3]. The results confirm once again the low incidence of astrovirus in gastroenteritis of children under 5 years old, in concordance with precedent studies made in Tunisia and Spain $[3,16]$.

HAstV-1 is the most common serotype identified in children in most countries where the astrovirus was analyzed [7, 8,18-19]. Previous Tunisian study also showed a predominance of the HAstV-1 (85.6 \%) over HAstV-3 (14.3\%) [10]. All the HAstVs obtained in 2003 were of serotype 1, while in 2004 the prevalence of HAstV-3 was relatively high (36\%). In 2005, all HAstVs found in Tunisia were HAstV-1 [10]. However, the two positive samples obtained in our study were HAstV-3. Different authors suggest a lack of heterotypic immunity between the different antigenic types of HAstV, which could be responsible for the changes in serotype distribution observed in consecutive years $[18,20]$.

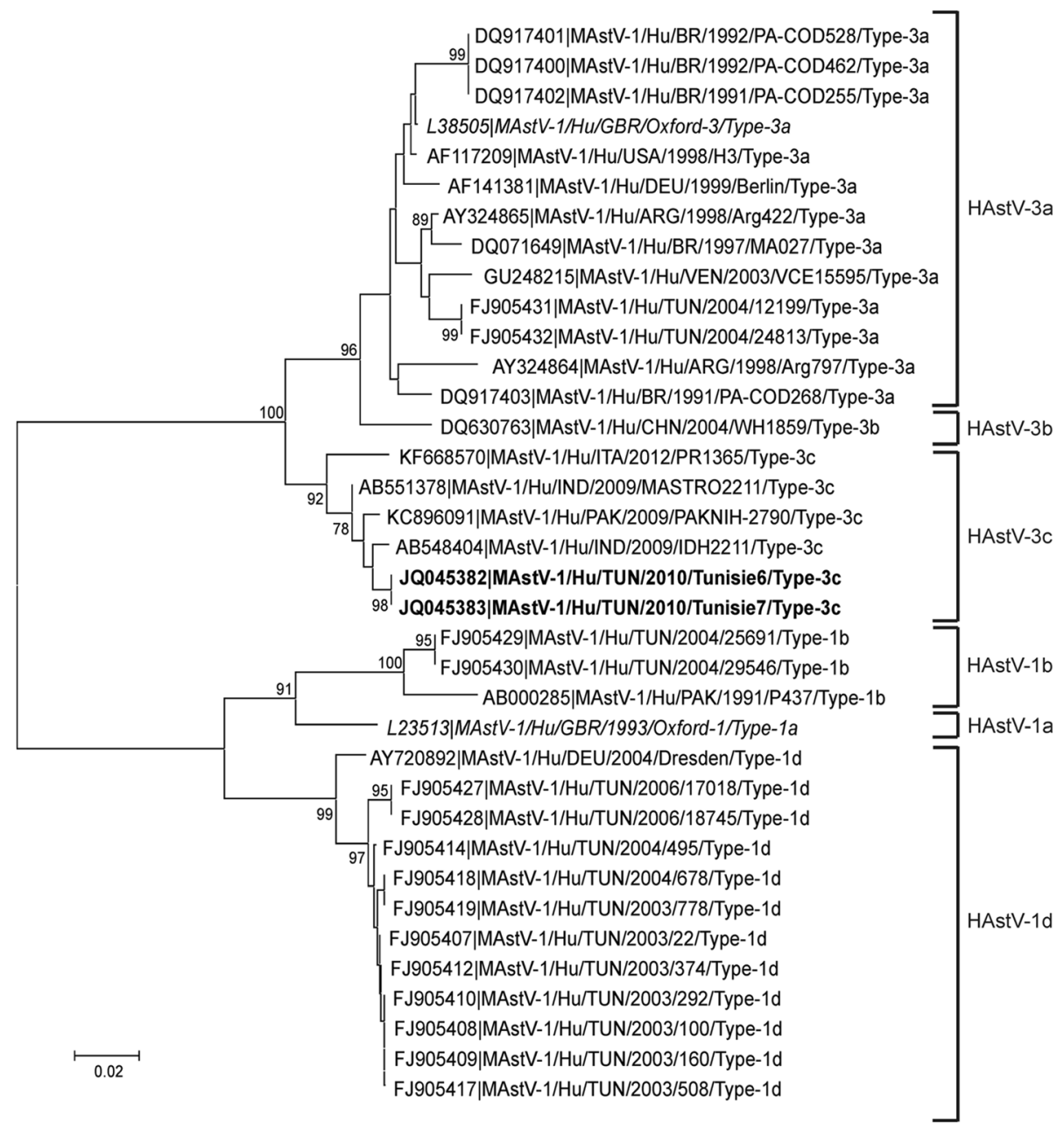

Fig. $3 \mathrm{NJ}$ phylogenetic tree obtained from sequences 
The phylogenetic analysis shows the occurrence of two different lineages of HAstV-3 in Tunisia. Strains obtained in 2004 [10] belong to lineage HAstV-3a, whilst our 2010 strains belong to lineage HAstV-3c. The Tunisian strains of the two lineages differ in $7.8 \%$ of nucleotides. Interestingly, many of the HAstV-3 recently reported are of the $3 c$ lineage $[15,21,22]$, suggesting that this lineage may be presently emerging.

PCR techniques are unable to differentiate between viral RNA associated to infectious or non-infectious particles. As shown in other studies, CaCO-2 cell culture combined with RT-PCR constitutes a reliable tool for evaluation of HAstV infectivity and increases PCR sensitivity as well $[23,24]$.

\section{Conclusions}

This study is part of a survey on the circulation of astroviruses in children hospitalized and/or outpatients for acute gastroenteritis in the Monastir region, Tunisia. The results show a low prevalence of astrovirus $(4 \%)$ in children younger than five years old. All genotyped samples belonged to lineage HAstV-3c. The results are in concordance with a lack of heterotypic immunity between the different antigenic types of HAstV. The phylogenetic analysis shows that Tunisian strains isolated in 2004 and 2010 belong to two different lineages and suggest that lineage HAstV-3c may have emerged and replaced HAstV-3a strains over time.

\section{Competing interests}

The authors declare that they have no competing interests.

\section{Authors' contributions}

Designed and performed the experiments: AM. Contributed with reagents/ materials/samples/analysis tools: NBSA, BM, SH, NG, LB. Analyzed the data: AM, NBSA, MLR. Wrote the paper: AM, JSC, SG, MA. All authors read and approved the final manuscript.

\section{Acknowledgements}

We gratefully thank the laboratory of Contagious Diseases and Biologically Active Substances team for technical assistance and Professor Albert Bosch from the Faculty of Biology (Barcelona University) for the revision of this paper.

\section{Author details}

'Laboratory of Contagious Diseases and Biologically Active Substances, LR99ES27, Faculty of Pharmacy, University of Monastir, Avicenne Street 5000, Monastir, Tunisia. ${ }^{2}$ Department of Microbiology, Faculty of Biology, University of Barcelona, Av. Diagonal 643, 08028 Barcelona, Spain. ${ }^{3} \mathrm{IRBIO}$ and Department of Animal Biology, Faculty of Biology, University of Barcelona, Av. Diagonal 643, 08028 Barcelona, Spain. ${ }^{4}$ Pediatric Department, University Hospital Fattouma Bourguiba, Monastir, Tunisia.

Received: 10 June 2015 Accepted: 12 January 2016

Published online: 21 January 2016

\section{References}

1. Walter JE, Mitchell DK. Astrovirus infection in children. Curr Opin Infect Dis. 2003:16(3):247-53.

2. Méndez E, Arias CF. Astroviruses. In: Knipe DM, Howley PM, Cohen Jl, Griffin DE, Lamb RA, Martin MA, Racaniello VR, Roizman B, editors. Fields virology. 5th ed. Philadelphia: Lippincott-Williams and Wilkins; 2007. p. 981-1000.
3. Bosch A, Pintó RM, Guix S. Human astroviruses. Clin Microbiol Rev. 2014; 27(4):1048-74.

4. Nguyen TA, Hoang L, le Pham D, Hoang KT, Mizuguchi M, Okitsu S, et al. Identification of human astrovirus infections among children with acute gastroenteritis in the Southern Part of Vietnam during 2005-2006. J Med Virol. 2008;80(2):298-305.

5. Matsui SM, Kiang D, Ginzton N, Che T, Geigenmüller-Gnirke U. Molecular biology of astroviruses: selected highlights. Novartis Found Symp. 2001;238: 219-33. discussion 233-236.

6. Gabbay YB, Leite JP, Oliveira DS, Nakamura LS, Nunes MR, Mascarenhas $J D$, et al. Molecular epidemiology of astrovirus type 1 in Belem, Brazil, as an agent of infantile gastroenteritis, over a period of 18 years (1982-2000): identification of two possible new lineages. Virus Res. 2007;129(2):166-74

7. De Grazia S, Martella V, Chironna M, Bonura F, Tummolo F, Calderaro A, et al. Nationwide surveillance study of human astrovirus infections in an Italian pediatric population. Epidemiol Infect. 2013;141(3):524-8.

8. Afrad MH, Karmakar PC, Das SK, Matthijnssens J, Ahmed F, Nahar S, et al. Epidemiology and genetic diversity of human astrovirus infection among hospitalized patients with acute diarrhea in Bangladesh from 2010 to 2012. J Clin Virol. 2013;58(4):612-8.

9. Mendez-Toss M, Romero-Guido P, Munguia ME, Mendez E, Arias CF. Molecular analysis of a serotype 8 human astrovirus genome. J Gen Virol. 2000:81:2891-7.

10. Sdiri-Loulizi K, Gharbi-Khelifi H, de Rougemont A, Hassine M, Chouchane S, Sakly N, et al. Molecular epidemiology of human astrovirus and adenovirus serotypes 40/41 strains related to acute diarrhea in Tunisian children. J Med Virol. 2009;81(11):1895-902

11. Noel JS, Lee TW, Kurtz JB, Glass RI, Monroe SS. Typing of human astroviruses from clinical isolates by enzyme immunoassay and nucleotide sequencing. Clin Microbiol. 1995:33(4):797-801.

12. Mitchell DK, Monroe SS, Jiang X, Matson DO, Glass RI, Pickering LK. Virologic features of an astrovirus diarrhea outbreak in a day care centre revealed by reverse transcriptase-polymerase chain reaction. J Infect Dis. 1995;172(6):1437-44.

13. Kimura M. A simple method for estimating evolutionary rate of base substitutions through comparative studies of nucleotide sequences. J Mol Evol. 1980:16(2):111-20.

14. Tamura K, Stecher G, Peterson D, Filipski A, Kumar S. MEGA6: molecular evolutionary genetics analysis version 6.0. Mol Biol Evol. 2013;30(12):2725-9.

15. Medici MC, Tummolo F, Martella V, Banyai K, Bonerba E, Chezzi C, et al. Genetic heterogeneity and recombination in type-3 human astroviruses. Infect Genet Evol. 2015:32:156-60.

16. Sdiri-Loulizi K, Gharbi-Khelifi H, De Rougemont A, Chouchane S, Sakly N, Ambert-Balay $\mathrm{K}$, et al. Acute infantile gastroenteritis associated with human enteric viruses in Tunisia. J Clin Microbiol. 2008:46(4):1349-55.

17. Sdiri-Loulizi K, Hassine M, Aouni Z, Gharbi-Khelifi H, Chouchane S, Sakly N, et al. Detection and molecular characterization of enteric viruses in environmental samples in Monastir, Tunisia between January 2003 and April 2007. J Appl Microbiol. 2010;109(3):1093-104.

18. Guix S, Caballero S, Villena C, Bartolome R, Latorre C, Rabella N, et al. Molecular epidemiology of astrovirus infection in Barcelona, Spain. J Clin Microbiol. 2002:40(1):133-9.

19. Jakab F, Walter JE, Berke T, Matson DO, Mitchell DK, Szucs G. Molecular characterization and sequence analysis of human astroviruses circulating in Hungary. FEMS Immunol Med Microbiol. 2003;39(2):97-102.

20. Naficy AB, Rao MR, Holmes JL, Abu-Elyazeed R, Savarino SJ, Wierzba TF, et al. Astrovirus diarrhea in Egyptian children. J Infect Dis. 2000;182(3):685-90.

21. Pativada M, Nataraju SM, Ganesh B, Rajendran K, Ramamurthy T, Ganguly S, et al. Emerging trends in the epidemiology of human astrovirus infection among infants, children and adults hospitalized with acute watery diarrhea in Kolkata, India. Infect Genet Evol. 2012;12(8):1685-93.

22 Alam MM, Khurshid A, Rana MS, Shaukat S, Sharif S, Angez M, et al. Serotype diversity of astroviruses in Rawalpindi, Pakistan during 2009-2010. PLoS One. 2013:8(4), e61667.

23. Mustafa H, Palombo EA, Bishop RF. Improved sensitivity of astrovirus-specific RT-PCR following culture of stool samples in CaCO2 cells. J Clin Virol. 1998; 11(2):103-7.

24. Grimm AC, Cashdollar JL, Williams FP, Fout GS. Development of an astrovirus RT-PCR detection assay for use with conventional, real-time, and integrated cell culture/RT-PCR. Can J Microbiol. 2004;50(4):269-78. 